\title{
Second and Fourth Harmonic Frequencies in Electric Field-Induced Liquid Crystal Reorientations
}

\author{
Xiangyi Nie, Haiqing Xianyu, Thomas X. Wu, \\ and Shin-Tson Wu \\ College of Optics and Photonics, University of Central Florida, \\ Orlando, Florida, USA
}

The second and fourth harmonics of low frequency electric field-induced liquid crystal reorientations are analyzed theoretically and confirming results observed experimentally. These transient dynamic optical responses manifest when the phase of a homogeneous cell is nearby $\mathrm{N} \pi$ or $\left(\mathrm{N}-\frac{1}{2}\right) \pi$, where $N$ is an integer. These ripples would influence the grayscales of liquid crystal devices if the driving electric field frequency is comparable to the liquid crystal response rate.

Keywords: electro-optical modulation; liquid crystal; second and fourth harmonics

\section{INTRODUCTION}

Nematic liquid crystal (LC) has been widely used for amplitude and phase modulations, such as displays [1,2], optical communications [3], adaptive lens [4-8], and tunable photonic devices [9,10]. The applied voltage can be either a square, sinusoidal or saw-tooth wave and the frequency can range from tens to a few kilo-hertz. To achieve grayscales, a bias voltage is applied [11]. Gray-to-gray switching usually occurs from a biased voltage to another biased voltage state. The LC response time is determined by the cell gap $(d)$, visco-elastic coefficient $\left(\gamma_{1} / \mathrm{K}\right)$, biased voltage, and applied voltage $[12,13]$. Various methods, such as thin cell gap [14-16], low viscosity materials [17], overdrive and undershoot voltage $[18,19]$, and elevated temperature [20], have been developed to improve the LC response time.

In this paper, we analyze the dynamic response of a homogeneous LC cell under a biased voltage condition. The sinusoidal electric field

Address correspondence to Shin-Tson Wu, College of Optics and Photonics, University of Central Florida, Orlando, FL 32816, USA. E-mail: swu@mail.ucf.edu 
frequency studied is around $f_{e} \sim 60 \mathrm{~Hz}$. In principle, square waves can be used too except that they contain high frequency components and are more difficult to analyze accurately. During analyses, we find that the second and fourth harmonic frequencies are generated depending on the biased voltage. Let us define $\delta_{0}$ as the effective phase retardation of the LC cell under a biased voltage, then the fourth harmonic frequency, i.e., $f_{o}=4 f_{e}$ is observed when $\delta_{0} \sim N \pi$ ( $N$ is an integer), and second harmonic frequency $f_{o}=2 f_{e}$ observed if $\delta_{0} \sim(\mathrm{N}-1 / 2) \pi$. To understand the underlying mechanisms, numerical simulation based on Finite Element Method (FEM) is performed to simulate the liquid crystal dynamic process. Confirming experimental results are obtained. These ripples will affect the grayscales of the LC device and should be considered carefully.

\section{THEORY}

Figure 1 depicts the device configuration used for our theoretical analysis. A homogeneous LC cell is sandwiched between two crossed polarizers. To obtain phase retardation, the LC director is oriented at $45^{\circ}$ with respect to the optic axis of the polarizer. A weak He-Ne laser $(\lambda=633 \mathrm{~nm})$ $\mathrm{nm}$ ) is used as the light source. The output transmittance is monitored by a computer controlled LabVIEW data acquisition system. The optical transmittance of this experimental setup can be expressed as [21]

$$
I=I_{0} \sin ^{2}\left(\frac{\delta_{0}}{2}\right)
$$

where $I_{0}$ is the incident light intensity and $\delta_{0}$ is the effective LC cell phase retardation, which is a function of cell gap, birefringence, voltage, and

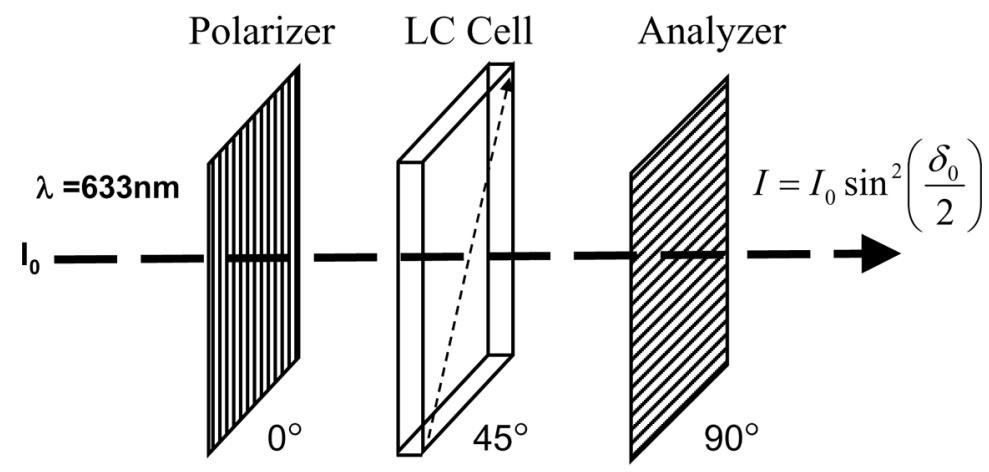

FIGURE 1 Experimental setup: a homogeneous liquid crystal cell is placed between a pair of crossed polarizer and analyzer. The cell director is oriented at $45^{\circ}$ with respect to the polarizer and analyzer, respectively. 
temperature. Because the applied sinusoidal driving voltage oscillates at a certain frequency, the LC directors could follow the transient external electric field dynamically. Thus a transient phase retardation shift $\Delta \delta$ exists around $\delta_{0}$, which results in a transient light modulation. The frequency and amplitude of the light modulation is dependent on the biased voltage.

Let us consider a small phase perturbation $(\Delta \delta)$ around $\delta_{0}$ and assume $\Delta \delta$ is much smaller than $\delta_{0}$, we could expand Eq. (1) using Taylor expansion and only keep the first and second order terms:

$$
\begin{aligned}
\frac{I}{I_{0}} & =\sin ^{2}\left[\frac{\delta_{0}+\Delta \delta}{2}\right]=\frac{1}{2}-\frac{1}{2} \cos \left(\delta_{0}+\Delta \delta\right) \\
& \approx \frac{1}{2}-\frac{1}{2}\left[\cos \delta_{0}-\sin \delta_{0} \cdot \Delta \delta-\frac{1}{2} \cos \delta_{0} \cdot \Delta \delta^{2}+\cdots\right] \\
& \approx \frac{1}{2}\left(1-\cos \delta_{0}\right)+\frac{1}{2} \sin \delta_{0} \cdot \Delta \delta+\frac{1}{4} \cos \delta_{0} \cdot \Delta \delta^{2}
\end{aligned}
$$

The transient phase $\delta(t)$ and phase change $\Delta \delta$ can be correlated to the LC director response time $\tau_{0}$ by the following equations [22]:

$$
\begin{gathered}
\delta(t) \approx \delta_{0} \exp \left(-2 t / \tau_{0}\right), \\
\Delta \delta(t) \approx \delta_{0}\left[1-\exp \left(-2 t / \tau_{0}\right)\right]
\end{gathered}
$$

which means that the phase response time is twice faster than the director's response time $\tau_{0}$.

If the phase retardation happens to be in the vicinity of $\delta_{0} \sim\left(N-\frac{1}{2}\right) \pi$, where $N$ is an integer, substituting Eq. (3b) to Eq. (2), we find

$$
\begin{aligned}
\frac{I}{I_{0}} & \approx \frac{1}{2}\left(1-\cos \delta_{0}\right)+\frac{1}{2} \sin \delta_{0} \cdot \Delta \delta+\frac{1}{4} \cos \delta_{0} \cdot \Delta \delta^{2} \\
& =\frac{1}{2}\left(1-\cos \delta_{0}\right)+(-1)^{N+1} \delta_{0}\left[1-\exp \left(-2 t / \tau_{0}\right)\right]
\end{aligned}
$$

From Eq. (4), the optical response time is $2 \mathrm{X}$ faster than the LC director response time $\tau_{0}$. However, when the phase retardation is $\delta_{0} \sim N \pi$, Eq. (2) can be reduced to

$$
\begin{aligned}
\frac{I}{I_{0}} & \approx \frac{1}{2}\left(1-\cos \delta_{0}\right)+\frac{1}{2} \sin \delta_{0} \cdot \Delta \delta+\frac{1}{4} \cos \delta_{0} \cdot \Delta \delta^{2} \\
& =\frac{1}{2}\left(1-\cos \delta_{0}\right)+(-1)^{N} \frac{\delta_{0}^{2}}{4} \cdot\left[1-\exp \left(-2 t / \tau_{0}\right)\right]^{2} \\
& =\frac{1}{2}\left(1-\cos \delta_{0}\right)+(-1)^{N} \frac{\delta_{0}^{2}}{4} \cdot\left[1-2 \exp \left(-2 t / \tau_{0}\right)+\exp \left(-4 t / \tau_{0}\right)\right]
\end{aligned}
$$




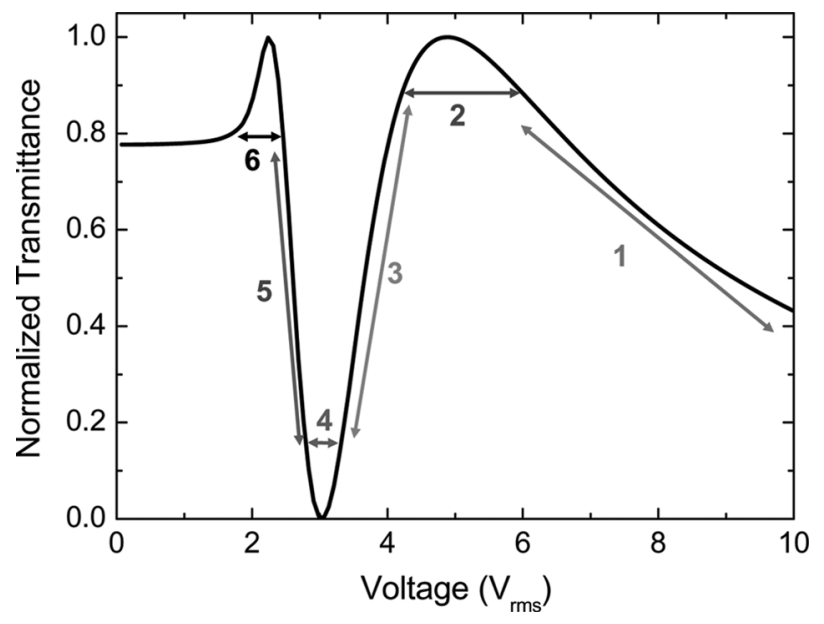

FIGURE 2 Voltage-dependent normalized transmittance of a homogeneous LC cell. Regions 2, 4, and 6 correspond to $\delta_{0} \sim N \pi$, and regions 1,3 , and 5 correspond to $\delta_{0} \sim\left(N-\frac{1}{2}\right) \pi$.

Under this condition, the optical response time can be up to $4 \mathrm{X}$ faster than $\tau_{0}$. Especially when $t / \tau_{0}$ is small, $I / I_{0}$ is mainly determined by the $\exp \left(-4 t / \tau_{0}\right)$ term because it decreases much faster than the $\exp \left(-2 t / \tau_{0}\right)$ term. These phenomena have been briefly mentioned in $[23,24]$, but no detailed derivations were given.

Figure 2 is a plot of voltage-dependent normalized transmittance of a typical homogeneous LC cell. When $\delta_{0} \sim N \pi$, the transmittance of the LC cell is either at a maximum (regions 2 and 6 ) or at a minimum (region 4). Under such a bias voltage condition, the fourth harmonic frequency is generated. The LC directors can closely follow the oscillation of the electric field, but at $4 \mathrm{X}$ higher frequency. If $\delta_{0} \sim\left(N-\frac{1}{2}\right) \pi$, i.e., the LC transmittance is near the regions 1,3 and 5 shown in Figure 2, the second harmonics takes place.

\section{RESULTS}

Experiments are conducted to validate the phase retardation dependent electro-optical modulation. FEM numerical simulation is also employed to simulate the transient phase and optical responses. The parameters of the LC mixture are listed as follows: elastic constants $K_{11}=11.7 \mathrm{pN}, K_{22}=8.8 \mathrm{pN}$, and $K_{33}=19.5 \mathrm{pN}$, dielectric anisotropy $\Delta \varepsilon=2.8$, birefringence $\Delta n=0.21, \gamma_{1}=0.25$ Pas, and the $\mathrm{LC}$ cell gap is $\mathrm{d}=4.99 \mu \mathrm{m}$. 
The finite element method (FEM) is a well recognized numerical simulation method [25]. A general purpose FEM computer program can be easily developed to rigorously analyze the problems where analytical solutions are difficult to obtain, such as calculating the transient phase shift. The numerical simulation of an LC device's dynamic behavior is based on the assumption that the system acts to minimize its free energy. The dynamic evolution of the LC director $n_{i}$ are provided by the analysis of the electric and elastic properties of the LC material, which is given by the Oseen-Frank free-energy equation,

$$
\begin{aligned}
F=\int_{v}\left[\frac{1}{2} K_{11}(\nabla \cdot \hat{n})^{2}+\frac{1}{2} K_{22}(\hat{n} \cdot \nabla \times \hat{n})^{2}\right. \\
\left.+\frac{1}{2} K_{33}(\hat{n} \times \nabla \times \hat{n})^{2}-\frac{1}{2} D \cdot E\right] d v,
\end{aligned}
$$

where $K_{11}, K_{22}, K_{33}$ are the three Frank elastic constants, and $\frac{1}{2} D \cdot E$ is the electric energy density of the free energy.

In the FEM program, we derive the dynamic equation for LC directors by considering the rotational viscosity $\gamma_{1}$ while ignoring the backflow effects,

$$
n_{i}^{\text {new }}=n_{i}^{\text {old }}-\frac{\Delta t}{\gamma_{1}}[F]_{n_{i}} .
$$

In each evolution step, $n_{i}$ is normalized as a unit, as shown in Eq. (8). Strong anchoring on the substrate surfaces is assumed in the simulation, and the boundary condition in the FEM simulation is described by prescribing $2^{\circ}$ pretilt angles on the LC cell surface planes. Once the LC director distribution is obtained, optical transmittance is calculated by the extended Jones matrix approach $[26,27]$.

As an example, $60 \mathrm{~Hz}$ sinusoidal driving voltage is used in our experiments and simulation, which is the typical frame rate of liquid crystal display. The amplitude of the driving voltage is adjusted to make $\delta_{0} \sim\left(N-\frac{1}{2}\right) \pi$ or $N \pi$.

As shown in Figure 3, the fourth harmonics is experimentally demonstrated. The peak-to-peak voltage $\left(\mathrm{V}_{\mathrm{pp}}\right)$ of the sinusoidal voltage is $14.87 \mathrm{~V}$, which is plotted in the lower trace of the figure. The corresponding optical response is plotted in the upper trace and its frequency is $240 \mathrm{~Hz}$, which is four times of the driving voltage. This result is consistent with our theoretical analysis, as shown in Eq. (5).

Numerical simulation results shown in Figure 4 also agree very well with the experiment. The simulation results show a transient phase shift $\Delta \delta$, and its peak-to-peak amplitude is $0.19 \pi$. The average phase shift $\delta_{0} \sim \pi$, which is in the region 2 of Figure 2 . The driving 


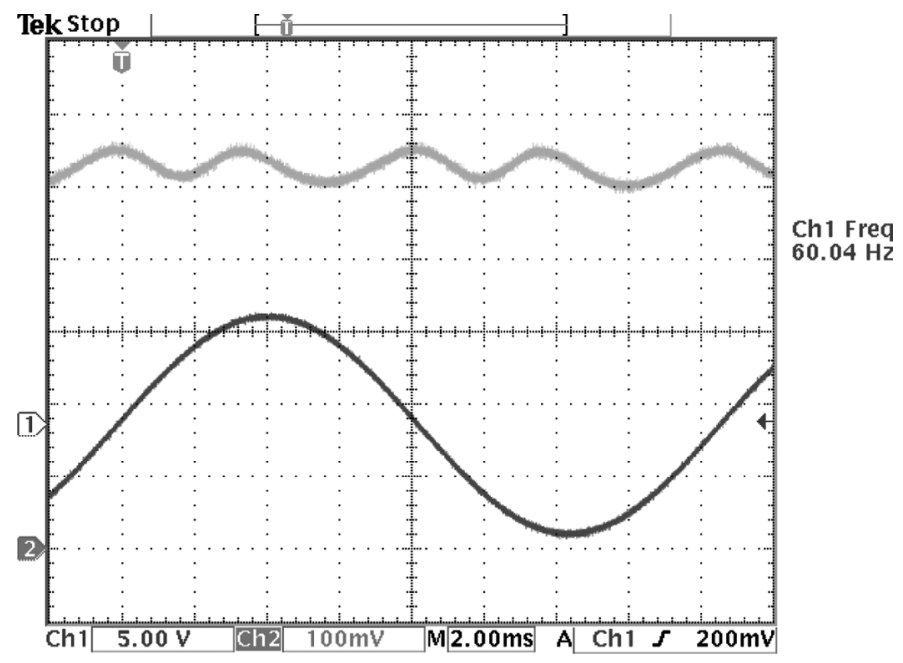

FIGURE 3 Experimental demonstration of $f_{o}=4 f_{e}$ at $f_{e}=60 \mathrm{~Hz}$. The applied voltage $\mathrm{V}_{\mathrm{pp}}=14.87 \mathrm{~V}$ and $\delta_{0}$ is around $1 \pi$.

voltage $\mathrm{V}_{\mathrm{pp}}$ is $13.8 \mathrm{~V}$, which is close to the $14.87 \mathrm{~V}$ employed in our experiments. The difference could originate from the finite anchoring energy and pretilt angle of the cell. Through simulations, we obtain a better understanding on the physical mechanisms responsible for the observed transient phase modulation and higher order harmonics in optical response.

Similarly, the second harmonics phenomenon is also experimentally observed, as shown in Figure 5. The applied voltage keeps an average phase retardation at $1.5 \pi$ and the peak-to-peak voltage is $\mathrm{V}_{\mathrm{pp}}=$ $11.81 \mathrm{~V}$. As discussed in the theory part, the frequency of optical response is $120 \mathrm{~Hz}$, which is two times of the driving frequency. Numerical simulation results shown in Figure 6 also confirm our experimental observation. In the simulation, $\mathrm{V}_{\mathrm{pp}}$ of the driving voltage is $11.80 \mathrm{~V}$, and the peak-to-peak amplitude of $\Delta \delta$ is $0.17 \pi$.

\section{DISCUSSION}

The observed transient phenomena are mainly determined by the dynamic driving voltage. The transient LC dynamic process is decided by the balance between the elastic and electric energy density, as represented by Eq. (7). The sinusoidal driving voltage is changing continuously and is applied on LC cells as a bias voltage. When the transient driving voltage decreases from its peak amplitude, LC directors 

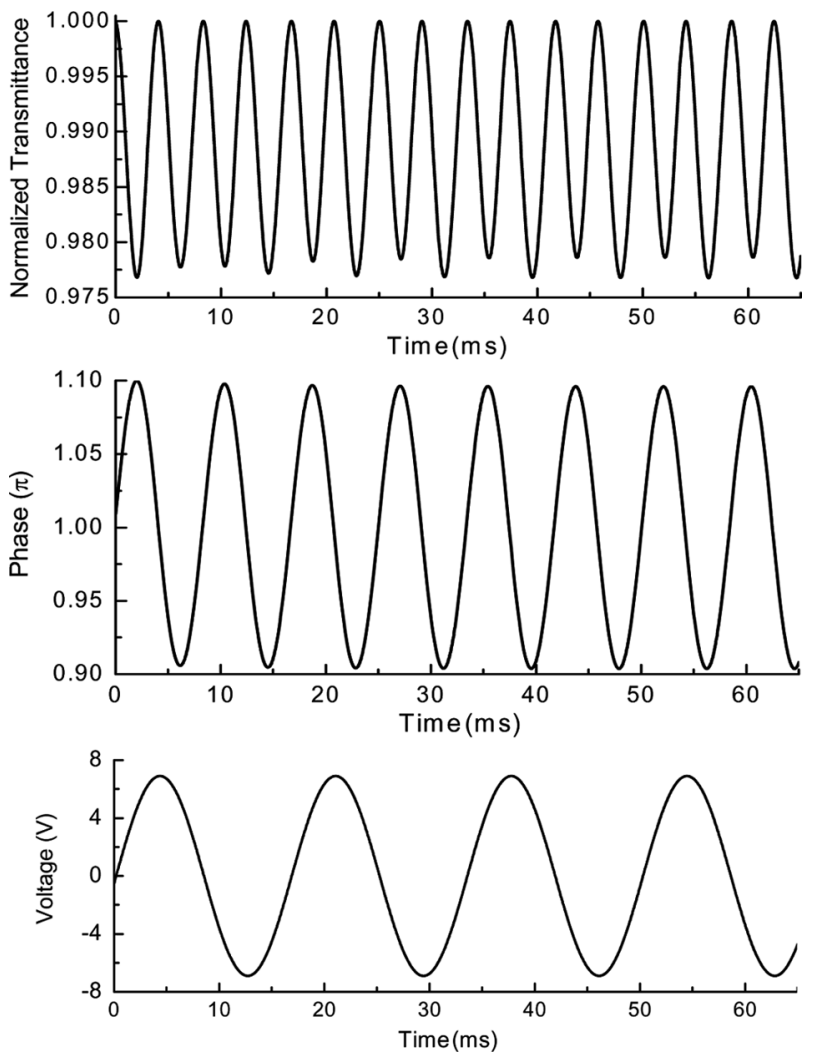

FIGURE 4 Simulation results of $f_{\mathrm{o}}=2 f_{p}=4 f_{e}$, at $f_{e}=60 \mathrm{~Hz}$. In the simulation, $\mathrm{V}_{\mathrm{pp}}=13.8 \mathrm{~V}$ and $\delta_{0} \sim \pi$, and the peak-to-peak amplitude of transient phase shift is $0.19 \pi$. The average phase retardation $\delta_{0}$ is $1.5 \pi$, and the transient phase shift is around region 2 in Figure 2.

relax accordingly as the bias voltage decreases. Once the decreasing cycle of voltage amplitude is finished, the driving voltage amplitude starts to increase. The increasing bias voltage reorients the LC directors back, even if the LC directors don't fully decay from the previous cycle yet. Therefore, the LC directors are consistently driven by the external electric field and they must follow the cycles of sinusoidal driving voltage. Since the sinusoidal driving voltage is changing continuously, a simple analytical solution is not available. Numerical simulation is used to solve Eq. (7) and calculate the transient phase shift. The simulation shows that, at a lower driving frequency, the longer period allows more time for LC directors' reorientation, which 


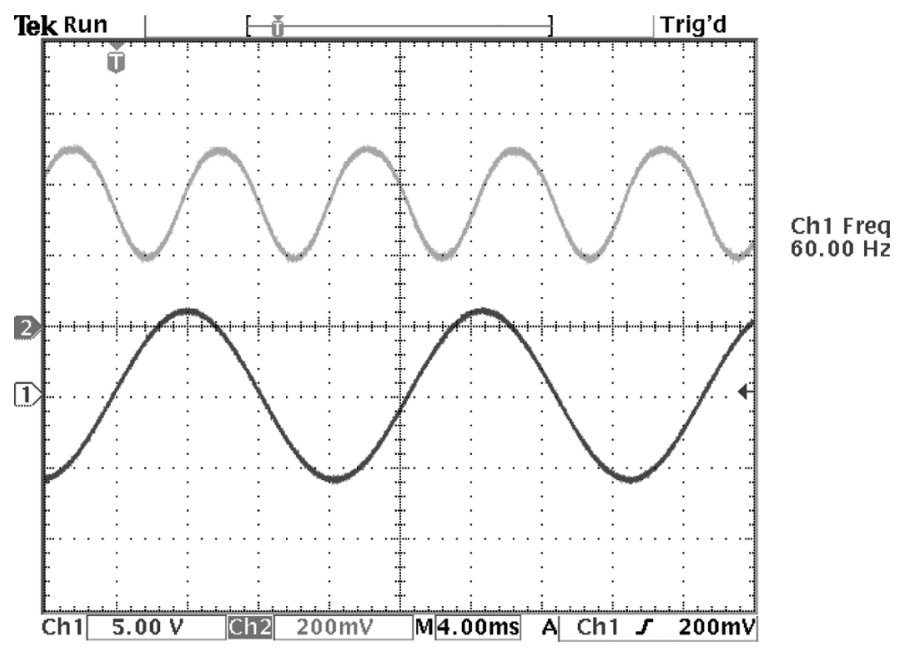

FIGURE 5 Experimental demonstration of $f_{o}=2 f_{e}$ at $f_{e}=60 \mathrm{~Hz}$. The peakto-peak voltage $\mathrm{V}_{\mathrm{pp}}=11.81 \mathrm{~V}$ and $\delta_{0} \sim 1.5 \pi$.
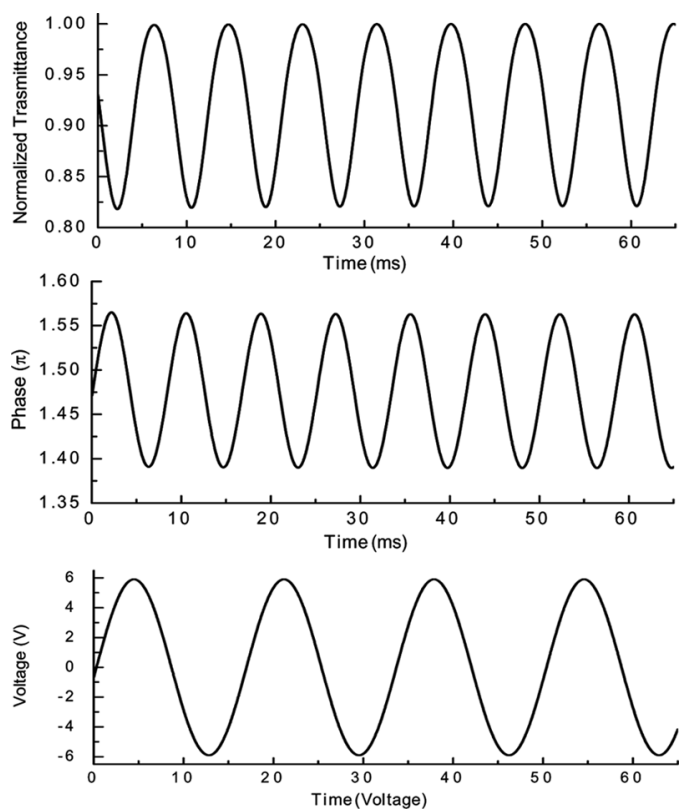

FIGURE 6 Simulation results of $f_{o}=2 f_{e}$, at $f_{e}=60 \mathrm{~Hz}$. In the simulation, $\mathrm{V}_{\mathrm{pp}}=11.80 \mathrm{~V}$ is used to keep $\delta_{0} \sim 1.5 \pi$, which is around region 3 in Figure 2. The $\Delta \delta$ peak-to-peak amplitude is $0.17 \pi$. 
leading to a larger $\Delta \delta$ and more significant LC cell transmittance fluctuation. The frequency of the driving voltage also influences $\Delta \delta$. The driving frequency of a thin-film-transistor (TFT) LCD is typically at $60-120 \mathrm{~Hz}$, thus, the transient optical fluctuation may influence the grayscales of the display device.

This phenomenon is general for nematic liquid crystal materials. Both our experiments and simulation show that a higher birefringence LC material causes a larger $\Delta \delta$ under the same driving condition. The voltage amplitude is another factor affecting $\Delta \delta$. According to our simulation larger voltage amplitude induces a more noticeable LC director deformation which, in turn, results in a larger $\Delta \delta$. And finally the total phase retardation $\left(\delta_{0}\right.$ and $\Delta \delta$ ) jointly determines the transient optical transmittance of the LC cell.

In this paper, we mainly discuss the sinusoidal driving voltage condition. However, this LC dynamic process can be generalized to other driving conditions, as long as a transient phase shift is induced by an external driving voltage. In our experiments, various voltage waveforms (rectangular and triangular) were used and similar results were observed.

\section{CONCLUSION}

The dynamics of liquid crystal electro-optical modulation is analyzed theoretically and validated experimentally. Under certain biased voltage conditions, the second and fourth harmonics are generated depending on the phase retardation of the LC cell studied. These second and fourth harmonic frequencies can affect the grayscales of a LC device. For applications which only require a small phase dithering, the fourth harmonic frequency which has $4 \mathrm{X}$ faster response time than the LC director's response time maybe useful.

\section{REFERENCES}

[1] Lueder, E. (2001). Liquid Crystal Displays, Wiley: New York.

[2] Yang, D. K. \& Wu, S. T., (2006). Fundamentals of Liquid Crystal Devices, Wiley: New York.

[3] McManamon, P. F., Dorschner, T. A., Corkum, D. L., Friedman, L., Hobbs, D. S., Holz, M., Liberman, S., Nguyen, H. Q., Resler, D. P., Sharp, R. C., \& Watson, E. A. (1996). Proc. of the IEEE, 84, 268.

[4] Sato, S. (1979). J. Appl. Phys., 18, 1679.

[5] Love, G. D., Major, J. V., \& Purvis, A. (1994). Opt. Lett., 19, 1170.

[6] Presnyako, V. V., Asatryan, K. E., \& Galstian, T. V. (2002). Opt. Express, 10, 865.

[7] Ren, H. \& Wu, S. T. (2002). Appl. Phys. Lett., 81, 3537.

[8] Ren, H. \& Wu, S. T. (2003). Appl. Phys. Lett., 82, 22. 
[9] Alkeskjold, T. T., Lægsgaard, J., Bjarklev, A., Hermann, D. S., Broeng, J., Li, J., \& Wu, S. T. (2004). Opt. Express, 12, 5857.

[10] Du, F., Lu, Y., \& Wu, S. T. (2004). Appl. Phys. Lett., 85, 2181.

[11] Wu, S. T. (1989). Appl. Opt., 28, 48.

[12] Jakeman, E. \& Raynes, E. P. (1972). Phys. Lett. A, 39, 69.

[13] Wang, H., Wu, T. X., Zhu, X., \& Wu, S. T. (2004). J. Appl. Phys., 95, 5502.

[14] Wu, S. T. \& Efron, U. (1986). Appl. Phys. Lett., 48, 624.

[15] Gauza, S., Zhu, X., Piecek, W., Dabrowski, R., \& Wu, S. T. (2007). J. Disp. Technol., $3,250$.

[16] Jiao, M., Ge, Z., Song, Q., \& Wu, S. T. (2008). Appl. Phys. Lett., 92, 061102.

[17] Geelhaar, T., Tarumi, K., \& Hirschmann, H. (1996). Soc. Inf. Display Tech. Digest, $27,167$.

[18] Wu, S. T. \& Wu, C. S. (1988). Appl. Phys. Lett., 53, 1794.

[19] Wu, S. T. \& Wu, C. S. (1989). J. Appl. Phys., 65, 527.

[20] Wu, S. T., Efron, U., \& Lackner, A. M. (1987). Appl. Opt., $26,3441$.

[21] Wu, S. T., Efron, U., \& Hess, L. D. (1984). Appl. Opt., 23, 3911.

[22] Wu, S. T. \& Wu, C. S. (1990). Phys. Rev. A, 42, 2219.

[23] Wu, S. T. (1986). J. Appl. Phys., 60, 1836.

[24] Blinov, L. M. \& Chigrinov, V. G. (1994). Electrooptic Effects in Liquid Crystal Materials, Springer: New York.

[25] Akin, J. E. (1994). Finite Elements for Analysis and Design, Academic Press: San Diego.

[26] Lien, A. (1990). Appl. Phys. Lett., 57, 2767.

[27] Ge, Z., Zhu, X., Wu, T. X., \& Wu, S. T. (2005). J. Opt. Soc. Am. A, 22, 966. 